\title{
Right Lower Quadrant of Abdomen
}

National Cancer Institute

\section{Source}

National Cancer Institute. Right Lower Quadrant of Abdomen. NCI Thesaurus. Code C151400.

The region of the abdomen that extends from the median plane to the right of the patient, and from the umbilical plane to the right inguinal lig ament. 